\title{
Perspectives on PET/MR Imaging: Are We Ready for Clinical Use?
}

$\mathbf{I}_{\mathbf{n}}$ cause it is difficult to question the primary role of PET/CT, PET/MR imaging has to demonstrate, at least to some extent, when its preference is justified despite its higher cost and more complex management- the topic of an article in this issue of The Journal of Nuclear Medicine (1).

The role of a diagnostic procedure is dependent on its comparative cost effectiveness versus alternative techniques that can answer the same questions. A diagnostic procedure may also become clinically useful if it makes an original contribution to the patient's management-for example, by integrating the diagnosis with further data that allow for an accurate prognosis or therapeutic planning. In justifying commercial and subsequent clinical

\section{See page 551}

interest in a new methodology, published results in the literature are to be relied on less than experience in clinical routine, where the available tools, expertise, and specific economic, organizational, and epidemiologic problems come into play. In determining the cost of the new methodology, the price of the single examination carries less weight than the economic impact on the entire clinical course, including savings deriving from a faster diagnosis, a shorter hospitalization, a more rigorous prognostic stratification, and a more effective therapeutic strategy. The choice of the most productive diagnostic technique has to be preceded by an evaluation of the associated risks and by elimination of the prejudices

Received Oct. 29, 2013; revision accepted Nov. 1, 2013.

For correspondence or reprints contact: Luigi Mansi, Medicina Nucleare, Seconda Università d Napoli. Piazza Miraglia, 2-80138 Napoli, Italy.

E-mail: luigi.mansi@unina2.it

Published online Feb. 20, 2014.

COPYRIGHT (C) 2014 by the Society of Nuclear

Medicine and Molecular Imaging, Inc.

DOI: 10.2967/jnumed.113.133405 with which radionuclide procedures are sometimes viewed.

A revolution in diagnostic imaging has been brought about by the advent of hybrid machines and their significantly improved accuracy, which was first applied in oncology. The success of PET/CT resulted in the complete disappearance of stand-alone PET. In contrast, SPECT/CT, despite its evident clinical superiority to stand-alone SPECT, has not displaced the latter. Evaluation of the cost effectiveness of PET/MR imaging appears to be more complex but also more intriguing.

Industries constructing hybrid machinery for humans are pursuing various commercial strategies. Philips Healthcare developed a system in which a time-of-flight (TOF) PET scanner and a 3-Tesla MR scanner are situated at opposite ends of a turntable in a single room. After the MR imaging study, the bed turns $180^{\circ}$ and slides the patient into the bore of the PET device; data fusion follows this sequential acquisition. GE Healthcare developed a 3-modality solution consisting of a PET/CT scanner and an MR scanner in adjacent rooms; the patient is transferred from one scanner to the other using a dockable table operating as a shuttle. In this approach, MR imaging is used mainly to complete the study by adding a further regional analysis of the territory previously scanned by PET/CT. Siemens Healthcare developed a true hybrid machine allowing a simultaneous MR and PET scan. The MR component is top-class, and the PET component has detectors (not allowing TOF) that do not affect the magnetic field. This scanner, which quickly became commercially available worldwide, was subsequently improved by the addition of a dedicated MR coil to allow guided biopsy of the breast and software permitting reliable correction of movement-particularly useful in children. More recently, GE Healthcare developed a true hybrid machine with a PET scanner using TOF detectors compatible with MR imaging; this device has not yet become commercially available.

Although there are no comparative studies, true hybrid machines not requiring repositioning are certainly less affected than sequential systems by the problem of possible misalignment. Furthermore, only simultaneous acquisition may allow the so-called 3-eye vision (2), in which the fusion of functional PET data with either morphostructural or functional MR data can mirror the real-time correspondence of MR and PET signals. Therefore, it is reasonable to think that true hybrid PET/MR imaging scanners will represent the real challenge to PET/CT. Conversely, the relevance of detectors allowing TOF acquisitions in hybrid machines has to be further evaluated. Although TOF PET has demonstrated technical advantages using traditional detectors, any superiority will probably become evident only when absolute quantitative data are routinely obtainable.

Overcoming the commercial primacy of PET/CT machines is difficult because of issues such as lower cost, faster performance, wider distribution, and experience in interpreting CT images, which is dependent on a radiologic formation privileging the morphostructural information. Nevertheless, several factors already support the preference of PET/MR imaging over PET/CT, at least in some fields.

The absence of ionizing radiation may significantly increase cost effectiveness in pediatric imaging or for nononcologic indications, such as inflammatory diseases. The lower radiation dose associated with PET/MR imaging will represent an advantage in patients with chronic diseases who must undergo periodic imaging to evaluate disease activity. PET/MR imaging might therefore be in higher demand for such subjects as those with Crohn disease, rheumatoid arthritis, granulomatosis, or fever of unknown origin. Similarly, the lower radiation dose could support the use of PET/MR imaging in oncologic patients undergoing multiple scans, particularly when such patients are young, as in the case of subjects being monitored for therapy response or with a high risk of relapse.

As a primary advantage, MR imaging frequently has better contrast than CT, allowing better visualization of soft tissues and bone marrow. Consequently, MR imaging may be superior to $\mathrm{CT}$ for evaluating territories with a heterogeneous tissue com- 
position further complicated by pathologic changes, such as hematologic malignancies with bone marrow involvement. In addition, either osteoblastic lesions or metastases limited to the bone marrow, negative on ${ }^{18}$ F-fluoride imaging but visible on MR imaging, could be individually delineated. A further advantage might be found in the evaluation of lesions localized to territories such as the brain, the pelvis, and the head and neck.

Unlike sequential methods, simultaneous image acquisition enables temporal coregistration of a dynamic PET acquisition and morphologic or functional MR data. In this way, the fused image may provide a variety of functional MR information, such as information on perfusion (microvessel density, vessel leakage), diffusion (cell density, microstructure), and metabolism (cell death, proliferation). Moreover, brain activity can be studied by functional MR imaging, and metabolism can be evaluated by MR spectroscopy. These uses require further acquisition time, which lengthens the duration of the whole study and would thus be feasible only for regional analyses, as in cerebral diseases, primary breast cancer, and chronic inflammation. Although the scanning time is significantly less for simultaneous PET/MR systems than for sequential PET/MR systems, neither type of system is yet competitive with the faster PET/CT acquisitions.

Because PET/MR imaging is better able to evaluate soft tissues and biologic components without significant changes in density, the technique might become preferred over PET/CT for patients with breast cancer, gynecologic tumors, colorectal cancer, diseases of the genitourinary system, cerebral diseases, and chronic inflammatory diseases. Conversely, the performance of PET/MR imaging would be worse in the study of lung, bone, and calcified lesions. Therefore, although at present pulmonary lesions up to $4 \mathrm{~mm}$ may be detected, some authors suggest the addition of chest CT if a PET/MR whole-body scan has negative findings in a patient with a high probability of lung metastases.

PET/MR is not able to have the measured attenuation correction feasible with PET/CT, but attenuation correction might be calculated on a postprocessed fused PET/MR image using a recent CT scan, or starting from MR maps, corrected with mathematic algorithms. The latter method could be considered reliable at the cranial level, because of the lack of movement and the possibility of more easily fitting a geometric model.

Although the clinical applications for PET/MR imaging described here, even when used only with conventional MR imaging sequences, may be hypothesized, future commercial distribution is difficult to anticipate. With the "third eye" being the most intriguing and original feature of PET/MR imaging, we believe that only the true hybrid systems having this feature will have a sufficient advantage over PET/CT to become commercially viable.

Unlike CT, MR imaging reveals not only differences in density but the dynamic molecular environment of the human being. Therefore, either morphostructural or pathophysiologic information can be obtained. The true hybrid PET/MR imaging systems that are presently commercially available already have the potential of 3-eye vision, although their scanning time is too long to allow for a complete wholebody study.

The study of brain diseases could be a good area for evaluating PET/MR imaging before clinical implementation, because the brain lacks movement and has a standard anatomic configuration not affected by variables such as fat or gases. Furthermore, technical problems concerning attenuation correction may be solved more easily, with the skull being easier to fit into a mathematic model. Moreover, because evaluation of cerebral diseases does not require a whole-body analysis, a study that also includes advanced MR procedures could more easily be performed. For example, a simultaneous evaluation of metabolism and the neural network could be performed, or the connection between radiotracer uptake and blood-brain barrier permeability could be investigated.

There are also some intriguing potential applications outside the brain. For example, PET/MR imaging could be used to acquire prognostic information through regional evaluation of a primary cancer or could assess the effect of biologic therapies in cancer patients. With functional MR imaging and its ability to evaluate neoangiogenesis and posttherapeutic modifications, interesting complementary results could be achieved using radiotracers beyond ${ }^{18} \mathrm{~F}-\mathrm{FDG}$, such as radiocholine in prostate cancer, DOTA-radiopeptides in neuroendocrine tumors, radiothymidine as a marker of tumor growth, and radiotracers evaluating hypoxia or angiogenesis. In this direction, diagnosis and therapy could be tailored through new and powerful tools that allow simultaneous analysis of angiogenesis and metabolism or of cellular growth and hypoxia, for example.

The real problem that PET/MR imaging has to overcome in its competition with $\mathrm{PET} / \mathrm{CT}$ is the need to demonstrate at least some degree of superiority in the evidencebased world of medicine, in which PET/CT dominates because of its wider distribution and lower cost and because medical staff are more skilled in its use.

Either nuclear physicians or radiologists have to better understand the significance of the pathophysiologic information that can be acquired by MR imaging. Without waiting for new experts who are able to evaluate the entire diagnostic content of fused PET/MR images, nuclear physicians need to educate themselves more widely and in greater depth not only on the morphostructural information provided by PET/MR imaging but also on the entire spectrum of functional information that can be gained.

\section{Luigi Mansi}

Second University of Naples Naples, Italy

\section{Andrea Ciarmiello \\ Sant'Andrea Hospital \\ La Spezia, Italy}

\section{REFERENCE}

1. Kuhn FP, Hüllner M, Mader CE, et al. Contrastenhanced PET/MR imaging versus contrast-enhanced PET/CT in head and neck cancer: how much MR information is needed? J Nucl Med. 2014;55:551-558.

2. Mansi L, Ciarmiello A, Cuccurullo V. PET/MRI and the revolution of the third eye. Eur J Nucl Med Mol Imaging. 2012,39:1519-1524. 